Research Article

\title{
Research on Ground Settlement Regularity of Soft Rock Tunnel under Ultrasmall Distance
}

\author{
Chunwu Zhang $\mathbb{D}^{1,2}$ Wei Hou $\mathbb{D}^{1},{ }^{1}$ Zongjun Sun $\mathbb{D}^{1},{ }^{1,3}$ and Li Gu $\mathbb{D}^{1}$ \\ ${ }^{1}$ Shandong Provincial Key Laboratory of Civil Engineering Disaster Prevention and Mitigation, \\ Shandong University of Science and Technology, Qingdao, Shandong 266590, China \\ ${ }^{2}$ China Railway Wuhan Survey and Design \& Institute Co., Ltd., Hubei, Wuhan 430074, China \\ ${ }^{3}$ Qingdao Ruihan Technology Group Co., Ltd., Qingdao, China \\ Correspondence should be addressed to Zongjun Sun; 3519808967@qq.com
}

Received 4 March 2021; Revised 8 May 2021; Accepted 27 May 2021; Published 7 June 2021

Academic Editor: Stefano Galassi

Copyright (C) 2021 Chunwu Zhang et al. This is an open access article distributed under the Creative Commons Attribution License, which permits unrestricted use, distribution, and reproduction in any medium, provided the original work is properly cited.

\begin{abstract}
During the tunnel construction under the road, the shallower the tunnel depth, the greater the effect of the surface settlement. Thus, to analyze the ground settlement caused by tunnel construction under ultrasmall distance, the research is based on a tunnel in Ningqiang County and uses numerical simulation and measurement to analyze the ground settlement and the effect of reinforcement measures. The research draws the following conclusions. For the ultrasmall distance tunnel under road construction, the combination of pipe shed and advanced small pipe grouting reinforcement measures inhibits the surface settlement. After the advanced small pipe is reinforced, the surface settlement value is reduced by about $25 \%$, and the reinforcement effect is more obvious after the increase of the large pipe shed. The surface settlement value is reduced by about $60 \%$. The surface settlement caused by the excavation of the circular soil accounts for about $50 \%-60 \%$ of the total settlement value, which is for the whole construction. The key processes, which involve the combination of reinforcement measures, focused on the suppression of surface settlement caused by the excavation of the circular soil. After the reinforcement measures, the variation of the settlement groove width was not obvious but the curvature and peak value of the settlement groove is reduced significantly.
\end{abstract}

\section{Introduction}

During the construction of the tunnel under passing the highway, the existing road pavement is susceptible to disasters, such as subsidence, cracking, and collapse caused by the disturbance of the tunnel excavation, which affects the normal operation of the existing traffic line [1]. The risk of pavement disaster is higher under ultraclose distances. Special reinforcement measures must be taken during the construction process to control ground loss and avoid damage to existing roads.

A large number of scholars at home and abroad have carried out corresponding research that seeks to avoid damage to the existing highway caused by the tunnel underpass construction and achieved certain results. Song et al. [2], based on the basic idea of random medium theory, improved the Parker method to obtain a simple and accurate surface settlement prediction method, which is more suitable for shallow tunnels. Chen et al. [3] established a threedimensional finite element calculation model to study the control effect of tunnel excavation under different excavation methods, different footages, and different support schemes. It is concluded that, in the construction of shallow tunnels, the excavation method should be selected as the double-side heading method, and the pre-reinforcement measures are to adopt double-layer large pipe roofs to reinforce. Li [4] analyzed the effects of excavation method, reserved bench length, advanced support, and tunnel burial depth on ground settlement based on the Harbin subway tunnel. Zhang et al. [5] used a three-dimensional finite difference model to simulate the advance grouting and grouting hardening of the shield tunnel and compared it 
with the field measurement data to analyze the lateral settlement, longitudinal settlement, and horizontal displacement of the ground. It is concluded that the lateral settlement occurs at about 1.5 times of the excavation diameter. Ruan and Sun [6] relied on the deformation of the roadbed and the ground during the shield construction process of the Wuhan Metro and concluded that a certain relationship between ground settlement and buried depth exists. The above research concluded that the shallower the tunnel burial depth, the greater the effect on ground settlement. Therefore, for the tunnel construction of ultrasmall distance under passing the highway, the risk is higher, and the current research is concentrated mostly on the underpass construction of shallow tunnels. Several studies have focused on the underpass construction of ultrashallow buried tunnels. Thus, the ground settlement caused by the ultrasmall distance underpass construction needs to be studied further.

This paper analyzes the law of ground settlement caused by the tunnel's ultrasmall distance underneath the highway construction based on a tunnel project in Ningqiang County. Using the method of combining numerical simulation and field measurement, the effects of the tunnel's ultrasmall distance underneath the highway construction are analyzed. The law of surface settlement was obtained, and the reinforcement effect of the adopted reinforcement measures was verified. Through research, construction experience and technical guidance were provided for the tunnel construction under ultrasmall distance through the highway.

\section{Project Overview}

2.1. Engineering Geology. The tunnel site is located in Ningqiang, Shanxi Province. The total length of the tunnel is $3471 \mathrm{~m}$, with an excavation height of $9.24 \mathrm{~m}$ and a span of $11.8 \mathrm{~m}$. It is a superlong road tunnel with a single tunnel and two-way driving. The tunnel underpasses the National Highway 108 at $\mathrm{K} 48+697$, and the underpass angle is about $80^{\circ}$. The layout is detailed in Figure 1. The buried depth of the tunnel at the underpass position is only $9 \mathrm{~m}$, which is less than 1 times that of the tunnel diameter. It is an ultrasmall underpass and a shallow tunnel. The width of the 108 country roadbed under the tunnel is $8.5 \mathrm{~m}$. The surrounding rock at the tunnel underpass is strong sandy shale, argillaceous structure, shale structure, broken rock mass, weathered joints, developed fissures, and poor stability of surrounding rock. It is grade $\mathrm{V}$ surrounding rock. The longitudinal section of the tunnel geology is shown in Figure 2.

2.2. Tunnel Design. The tunnel support adopts a composite lining structure, as shown in Figure 3, and the supporting parameters are listed in Table 1.

The tunnel arch adopts a combination of conventional reinforcement of advanced small pipe grouting and special reinforcement of the large pipe shed to control the surface settlement and improve the stability of the surrounding rock. The diameter of the advanced small pipe is $42 \mathrm{~mm}$, the length is $L=5 \mathrm{~m}$, the erection angle is $7^{\circ}$, and the

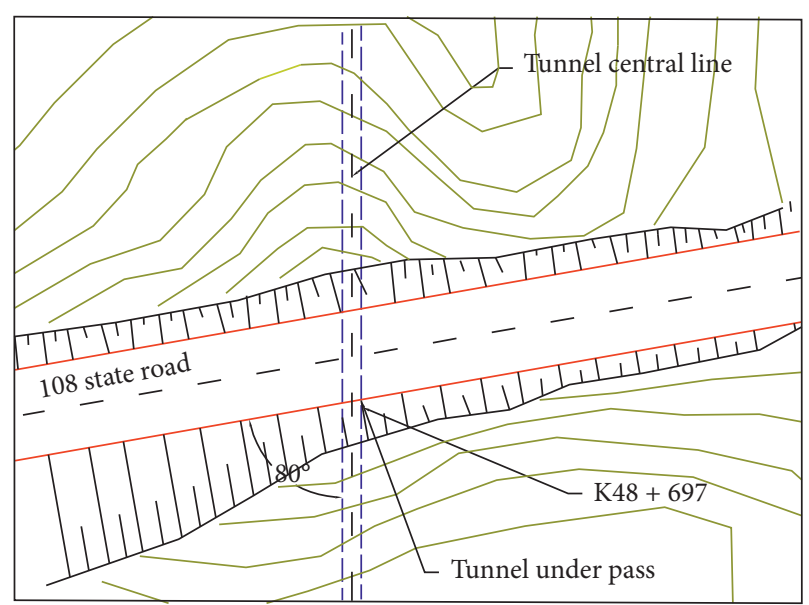

Figure 1: Tunnel under the National Road 108 Plan.

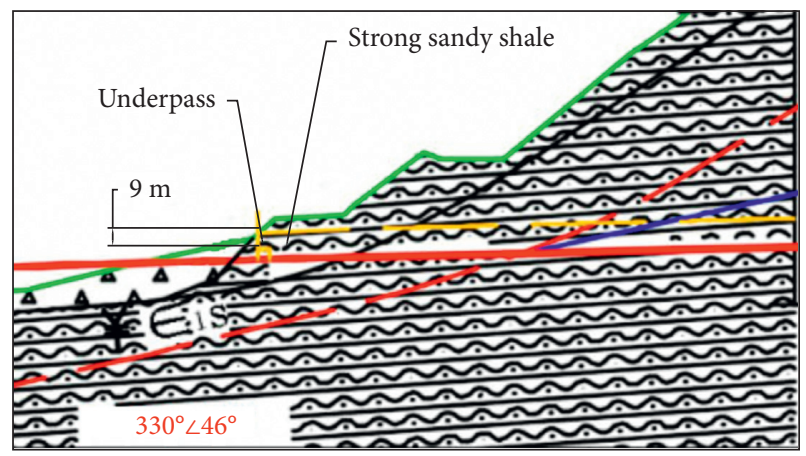

Figure 2: Geological profile of the tunnel.

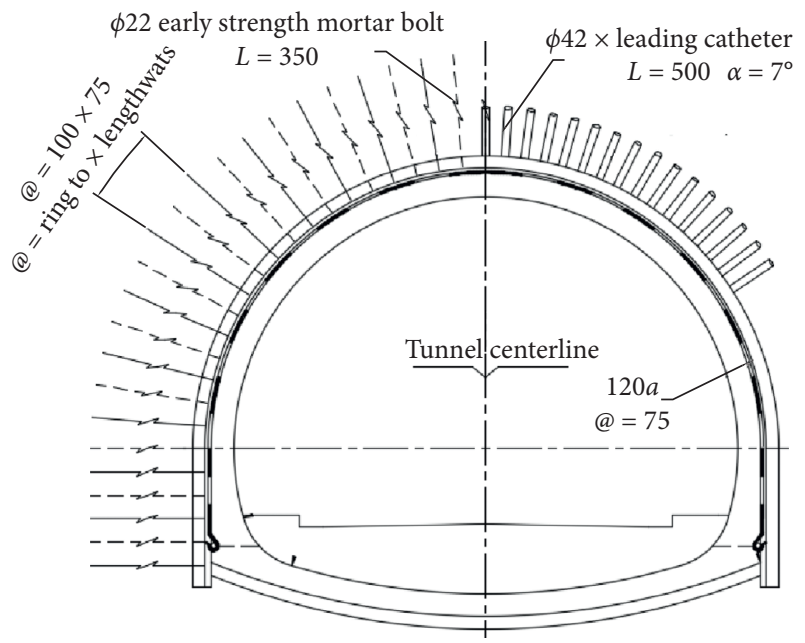

Figure 3: Design drawing of the tunnel support structure.

circumferential spacing is $0.4 \mathrm{~m}$. After the small pipe is completed, the concrete grout is poured. The large pipe shed adopts $\varphi 89 \mathrm{~mm} \times 6 \mathrm{~mm}$ steel pipes with a length of $30 \mathrm{~m}$ and an external insertion angle of $2^{\circ}$.

The reserved core soil method is used to construct the tunnel underneath the existing highway, as shown in Figure 4. Before excavating the tunnel, a large pipe roof and advanced small pipe grouting are used for advanced 
TABle 1: Parameters of tunnel support.

\begin{tabular}{lccccc}
\hline Materials & Shotcrete & Shape steel & Rock bolt & Secondary lining concrete & Rebar \\
\hline Specification & C20 & I20a @75 cm & $\varphi 22 \mathrm{~mm}$ Mortar Anchor $L=3.5 \mathrm{~m}$ & C25 & $\varphi 22 @ 250 \mathrm{~mm}$ \\
\hline
\end{tabular}

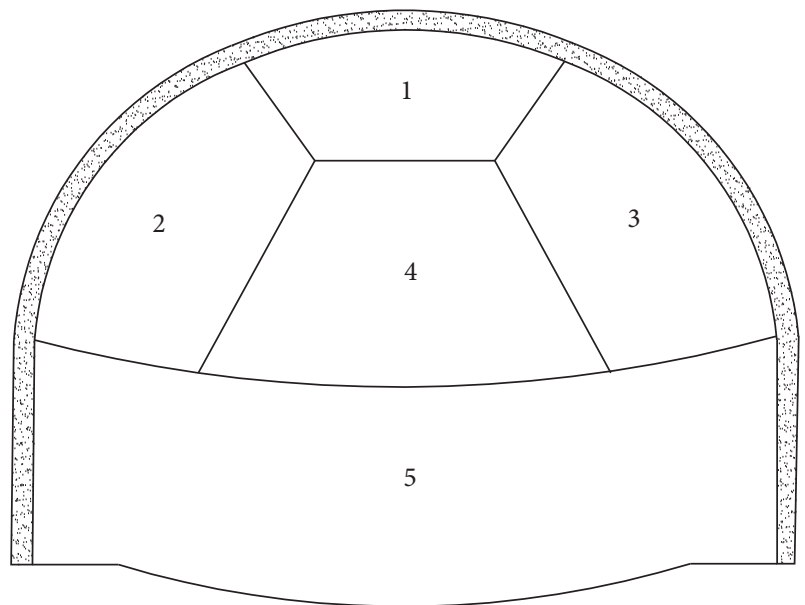

FIgURE 4: The construction process of reserved core soil.

reinforcement. Subsequently, the upper ring soil was excavated, then the core soil was excavated, and finally, the lower step was excavated. The core soil is excavated after $3 \mathrm{~m}$ of ring soil excavation, and the lower bench excavation is carried out after $5 \mathrm{~m}$ of core soil excavation. The initial support will be implemented after the excavation of each part is completed. After the initial support is closed into a ring of $10 \mathrm{~m}$, the second lining will be constructed.

\section{Numerical Analysis of Surface Subsidence}

The ground is affected by the disturbance of tunnel excavation during the construction process, and settlement control is difficult because of the ultrasmall underpass of the tunnel. In this regard, the tunnel arch was reinforced with a combination of advanced small pipe grouting and a large pipe shed. To analyze the effects of reinforcement measures, numerical simulation methods are used to study the surface settlement laws under three conditions: no reinforcement measures, advanced small pipe grouting reinforcement, and advanced small pipe grouting combined with large pipe shed reinforcement.

\subsection{Numerical Model and Calculation Parameters.} FlAC3.0 is used to establish a numerical model. The subgrade, lining, and surrounding rock are all made of solid elements. The model size is $65 \mathrm{~m} \times 50 \mathrm{~m} \times 20 \mathrm{~m}$. Horizontal constraints are imposed on the front-posterior left-right boundary, and vertical constraints are imposed on the bottom boundary distance, as shown in Figure 5.

Preconsolidation is a key link in controlling ground subsidence $[7,8]$. For this, the numerical model focuses on the preconsolidation of tunnels. The large pipe shed adopts the pile unit, and the small pipe adopts the beam unit simulation, as shown in Figures 6 and 7.
The equivalent elastic modulus of the pipe roof and grouting small pipe is equivalently converted according to the concrete-filled steel tube:

$$
E=\frac{E_{1} I_{1}+E_{2} I_{2}}{I_{1}+I_{2}},
$$

where $E$ is the equivalent elastic modulus, $E_{1}$ and $E_{2}$ are the elastic modulus of the cement slurry and pipe shed (small duct), respectively, and $I_{1}$ and $I_{2}$ are the inertia moment of the cement slurry and pipe shed (small duct), respectively.

The surrounding rock conforms to Mohr-Coulomb's law, and the supporting structure conforms to the elastic theoretical hypothesis $[9,10]$. The calculation parameters of the surrounding rock and supporting structure are shown in Table 2.

The tunnel beneath the highway section uses the provided core soil method. The pipe shed is erected one time before the tunnel excavation, and the advanced small pipe grouting is gradually installed before each excavation. In the tunnel, the upper ring soil is excavated first, and the reserved core soil excavation lags behind the ring soil excavation by $3 \mathrm{~m}$. The lower bench excavation lags behind the reserved core soil excavation by $5 \mathrm{~m}$, and each excavation length is $1 \mathrm{~m}$, as shown in Figure 8. After each step of excavation, the initial support is applied, and the second lining is applied after the excavation of $10 \mathrm{~m}$ down the steps.

3.2. Numerical Calculation of Surface Settlement. Numerical simulation is used to analyze the law of surface settlement caused by tunnel excavation under three working conditions: no reinforcement measures, advanced small pipe grouting reinforcement, and large pipe roof and advanced small pipe grouting reinforcement. First, the calculation results of the maximum cumulative settlement of the three groups of working conditions after annular soil excavation, reserved core soil excavation, and lower bench excavation are shown in Figure 9.

A comparison of the maximum surface settlement values under the three working conditions shows that the maximum surface settlement values after the reinforcement measures are reduced significantly. After adopting advanced small pipe reinforcement, the maximum surface settlement value is reduced by about $25 \%$ compared with the maximum surface settlement value without reinforcement measures. The maximum surface settlement value is $24.1 \mathrm{~mm}$, which still exceeds the alarm value of $24 \mathrm{~mm}$ [11]. After adopting the combined reinforcement measures of large pipe roof and advanced small pipe grouting, the maximum surface settlement value is reduced by about $60 \%$ compared with the maximum surface settlement value without reinforcement measures. The maximum surface settlement value is $12.2 \mathrm{~mm}$, which meets the safety performance requirements. Compared with the advanced small pipe grouting 


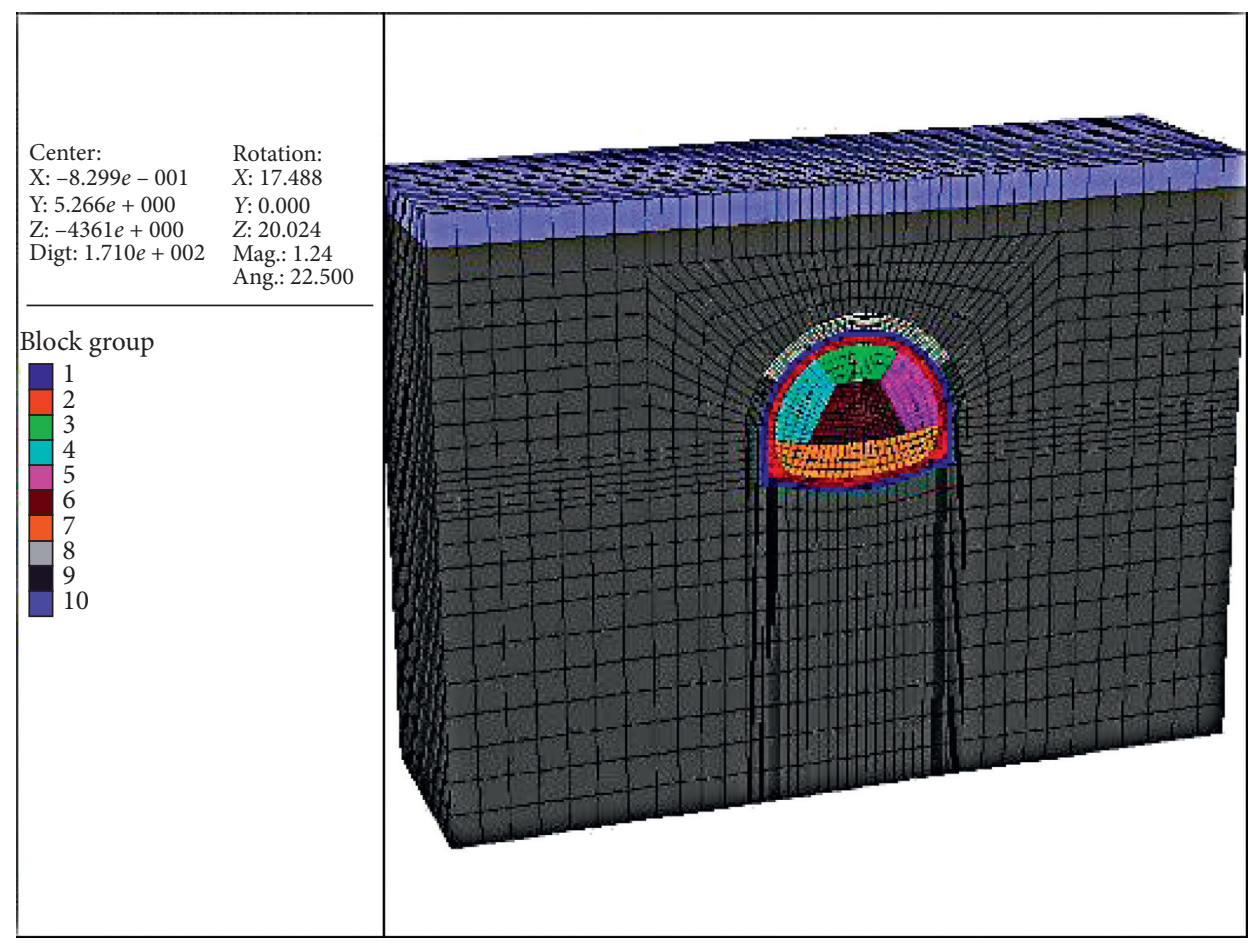

FIGURE 5: Underpass numerical model of the tunnel.

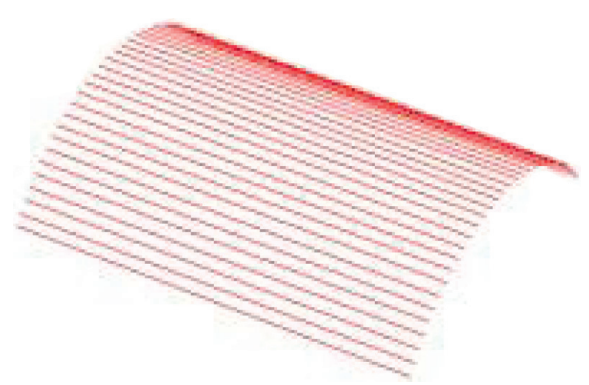

Figure 6: Pile unit of the pipe roof.

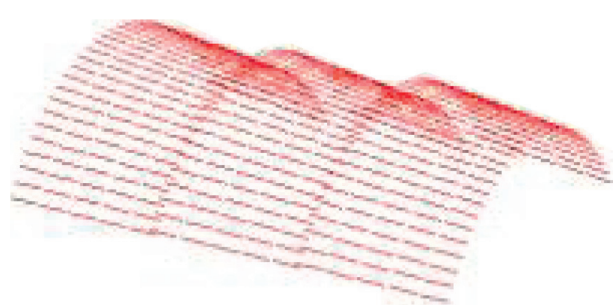

Figure 7: Beam unit of a small catheter.

reinforcement, the reinforcement effect is more obvious after the large pipe shed is added.

The surface subsidence values caused by each excavation stage are compared and analyzed as listed in Table 3.

The above table shows that, during tunnel excavation, the stage with the largest surface settlement amplitude is when the annular soil is excavated. For the reinforced and unreinforced strata, the surface settlement caused by the excavation of the annular soil is relatively large, and the decrease is about $50 \%$ to $60 \%$ of the total settlement value. It is a key process of the entire construction phase, and key monitoring should be carried out during the construction process. The excavation of the core soil and the lower bench caused relatively small surface settlements, and their respective declines accounted for about $23 \%$ to $35 \%$ and $15 \%$ to $20 \%$ of the total settlement value.

Compared to the surface settlement value caused by each process under the combined reinforcement of the pipe shed and the advanced small pipe with the value of the surface settlement value without reinforcement measures after the excavation is completed, the ground settlement with combined reinforcement measures is $18.5 \mathrm{~mm}$ lower than the ground settlement without reinforcement measures. After the excavation of the annular soil, the ground settlement with combined reinforcement measures is reduced by $12.7 \mathrm{~mm}$ compared with the ground settlement without reinforcement measures. Hence, the combined reinforcement measures focused on restraining the surface settlement value caused by the excavation of the annular soil.

The upper surface settlement trough caused by each construction process of the three working conditions is compared, and the comparison results are shown in Figures 10 and 11.

Figures 10-12 show that the tunnel is gradually excavated, and the upper surface settlement trough increased to a certain extent, but the width increase is not obvious. Because of the shallow buried depth of the tunnel, the settlement groove width caused by tunnel excavation is small at about three times the buried depth of the tunnel. However, the curvature and peak value of the settlement trough is relatively large, and this type of tunnel construction is very 
TABle 2: Parameter of numerical calculation.

\begin{tabular}{lccccc}
\hline Items & Weight $\left(\mathrm{kN} \cdot \mathrm{m}^{-3}\right)$ & Elastic modulus $(\mathrm{GPa})$ & Poisson ratio & Cohesive force $(\mathrm{kPa})$ & Internal friction angle \\
\hline Wall rock & 18 & 1 & 0.4 & 100 & 20 \\
Condulet/Pipe shed & 33 & 93 & 0.21 & - & - \\
Reinforcement area & 21 & 1.4 & 0.35 & - & 350 \\
Preliminary bracing & 25 & 28 & 0.2 & - & - \\
Secondary lining & 25 & 29.5 & 0.2 & - \\
\hline
\end{tabular}

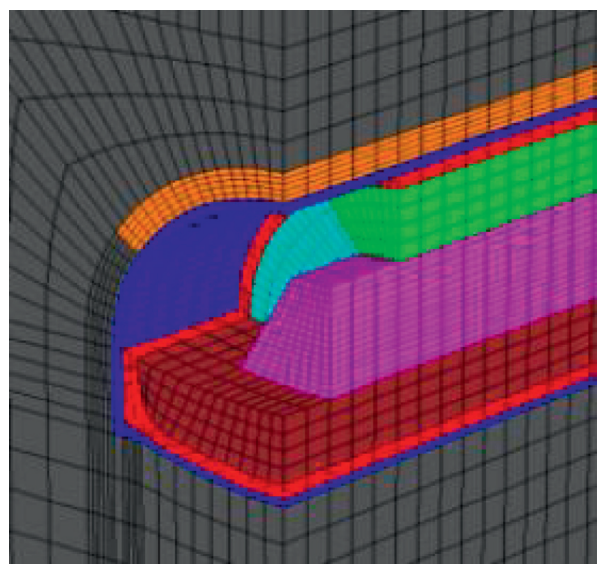

FIGURE 8: Simulation of the construction method of reserved core soil.

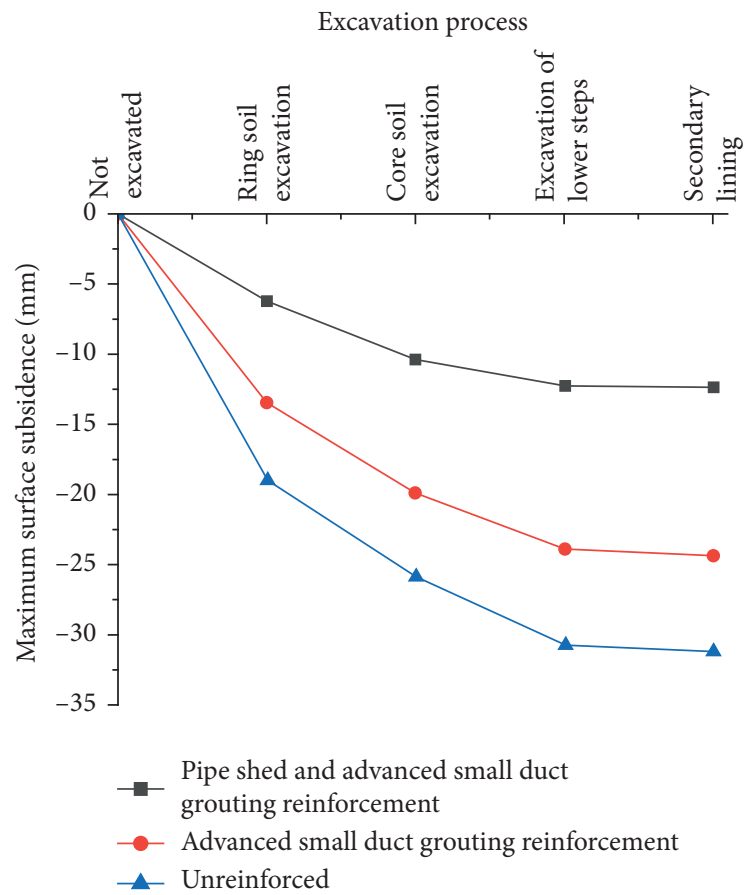

FIgURE 9: Comparison of maximum accumulated surface settlement. 
TABLE 3: Comparison of surface settlement values caused by each process.

\begin{tabular}{lccc}
\hline The excavation phase & $\begin{array}{c}\text { Pipe-shed small conduit combination } \\
\text { reinforcement }(\mathrm{mm})\end{array}$ & $\begin{array}{c}\text { Advance grouting reinforcement of small } \\
\text { conduit }(\mathrm{mm})\end{array}$ & $\begin{array}{c}\text { Unreinforced } \\
(\mathrm{mm})\end{array}$ \\
\hline Annular excavation & -6.1 & -13.4 & -18.9 \\
Core excavation & -4.2 & -6.4 & -6.8 \\
Lower step excavation & -1.8 & -4 & -4.9 \\
Excavation causes cumulative & -12.1 & -23.8 & -30.6 \\
settlement & & & \\
\hline
\end{tabular}

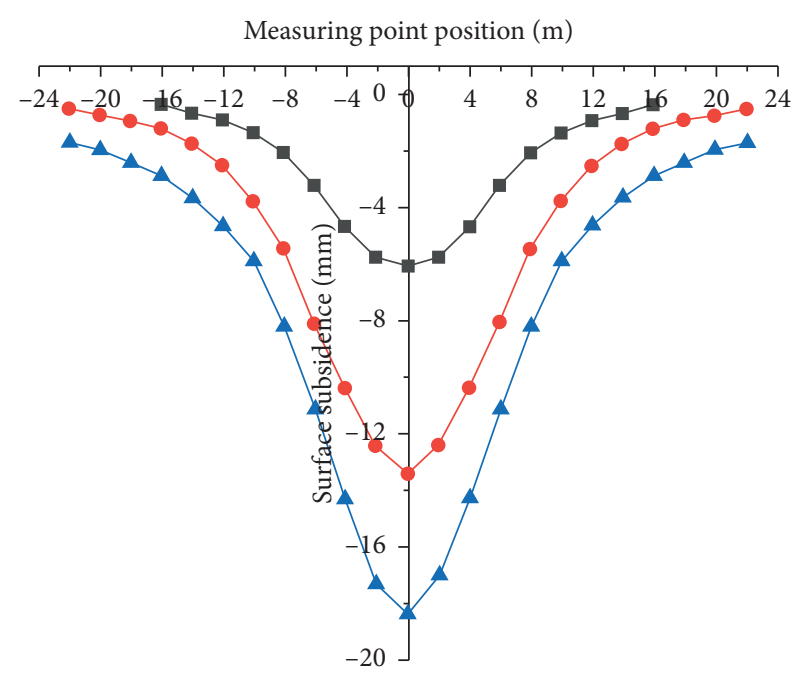

- - Pipe shed and advanced small duct grouting reinforcement

- Advanced small duct grouting reinforcement

- $\_$Unreinforced

Figure 10: Distribution of surface settlement trough after the excavation of annular soil.

Measuring point location (m)

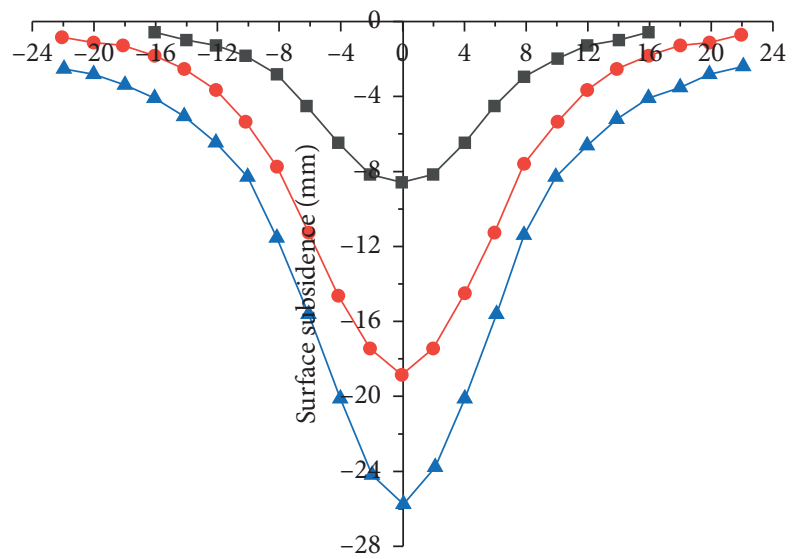

- Pipe shed and advanced small duct grouting reinforcement

- Advanced small duct grouting reinforcement

-1_ Unreinforced

Figure 11: Distribution of surface settlement trough after the excavation of core soil.

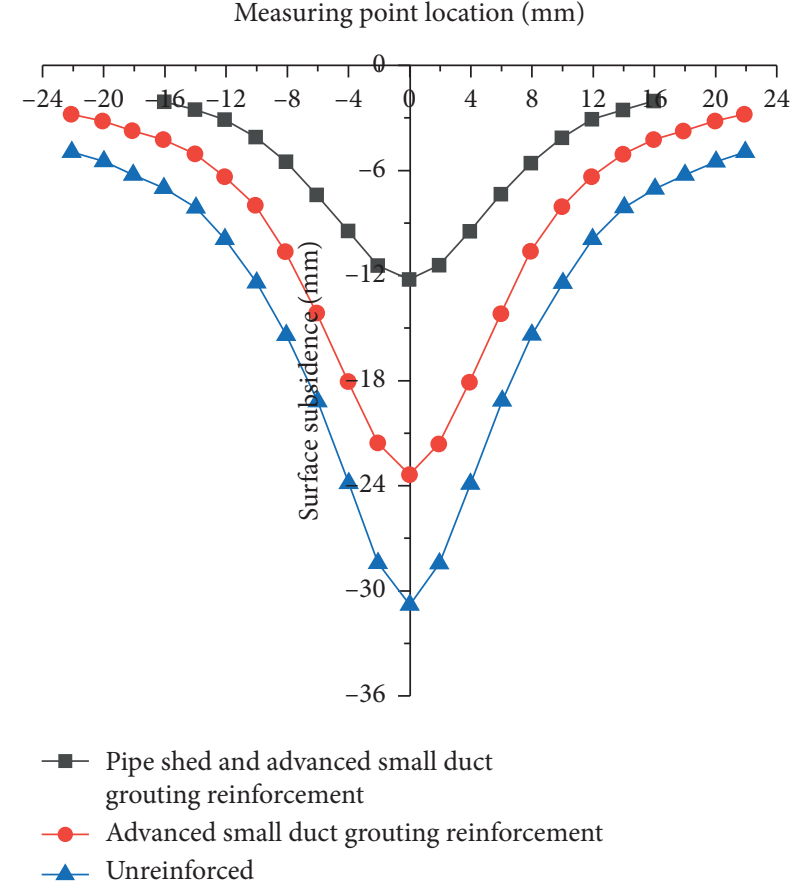

Figure 12: Distribution of surface settlement trough after the excavation of lower bench soil.

prone to collapse. The settlement groove width does not change significantly with the implementation of various reinforcement measures. However, the curvature and peak values of the settlement tank have been reduced significantly. The combined reinforcement measures of the pipe roof and the advanced small pipe grouting can significantly reduce the maximum settlement value, thereby reducing the possibility of tunnel collapse.

\section{Surface Subsidence Monitoring Site}

During the construction of the tunnel underneath the highway, the ground settlement was monitored on site, and the location of the surface measurement point is shown in Figure 13. With the increase of excavation footage, the settlement measured on site is shown in Table 4, and the time history curve of the ground settlement with the tunnel excavation and the distribution of the ground settlement trough were obtained, as shown in Figures 14 and 15. 


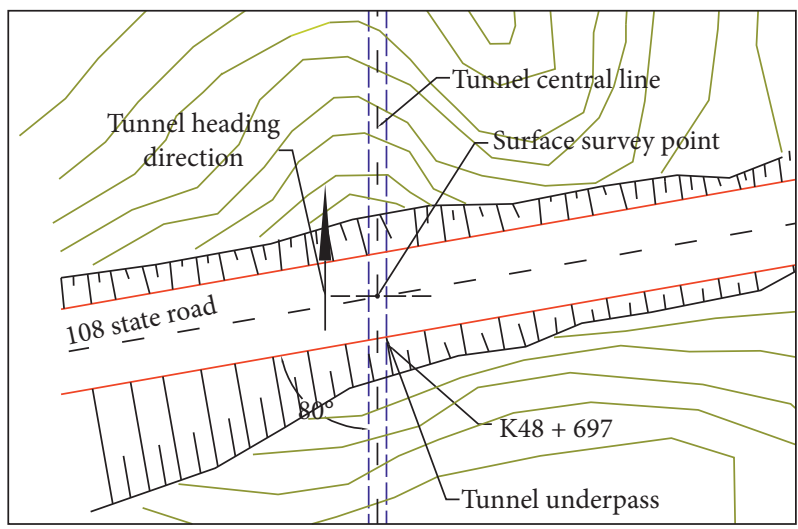

FIGURE 13: Location of surface survey points.

TABLE 4: Increase the settlement measured on site with the excavation footage.

\begin{tabular}{lccccccccccc}
\hline Excavation footage $(\mathrm{m})$ & 2 & 4 & 6 & 8 & 10 & 12 & 14 & 16 & 18 & 20 & 22 \\
Settlement $(\mathrm{m})$ & 4.47 & 1.43 & 2.11 & 0.88 & 0.16 & 1.58 & -0.32 & 0.10 & 0.20 & 0.07 & -0.04 \\
\hline
\end{tabular}

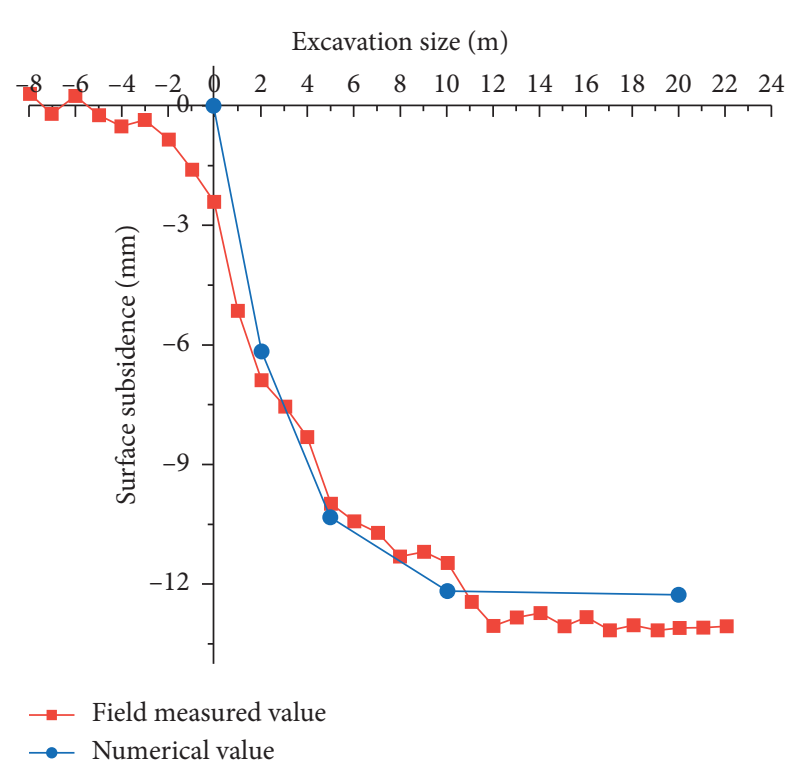

FIGURE 14: Time history curve of surface settlement caused by tunnel excavation.

The figures show that the on-site monitoring data of ground settlement are close to the law as presented by the numerical simulation results, which verifies that the numerical simulation results can effectively provide support for the determination of reinforcement measures for tunnel underpass construction. During the entire construction process, the maximum surface settlement value is $13.11 \mathrm{~mm}$, which is less than the safety control standard of $30 \mathrm{~m}$ in the specification. After taking pipe roof reinforcement measures and using advanced small pipe grouting, surface subsidence caused by the tunnel dropped to a low level to meet the safety requirements. For the construction of ultrasmall tunnels underneath the highway, the reinforcement measures of pipe shed and leading small pipe grouting have a good effect on controlling surface settlement.

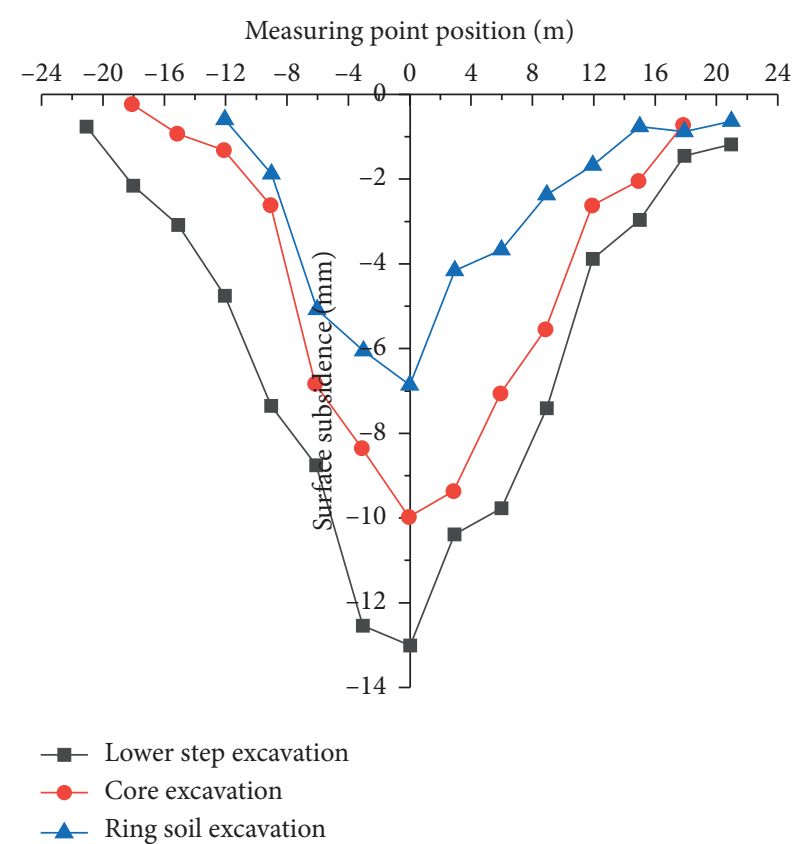

Figure 15: Distribution of surface settlement trough after the excavation of field measurement.

\section{Conclusion}

The shallower the tunnel buried depth, the greater the effect on surface settlement. The risk of road disasters is higher for shallow tunnels that cross the highway at a very close distance. Thus, to analyze the law of surface settlement caused by the construction of the tunnel under the road through the ultrasmall distance, the study relied on a tunnel in Ningqiang County and used the method of combining numerical simulation and field measurement to analyze the law of ground settlement caused by the construction of the tunnel under the road at the ultrasmall distance. The reinforcement effect of pipe shed and advanced small duct grouting is 
discussed. The research has drawn the following conclusions:

(1) After adopting the combined reinforcement measures of pipe shed and advanced small pipe grouting, the surface settlement caused by the tunnel is at a relatively low level, which meets the safety requirements. For the construction of ultrasmall tunnels underneath the highway, the reinforcement measures of pipe shed and leading small pipe grouting have a good effect on controlling surface settlement.

(2) After adopting the advanced small pipe reinforcement, the maximum surface settlement value is reduced by about $25 \%$ compared with the case without reinforcement measures. After adopting the combined reinforcement measures of the large pipe shed and the advanced small pipe grouting, the maximum surface settlement value is reduced by about $60 \%$ compared with the absence of reinforcement measures. Compared with the advanced small pipe grouting reinforcement, the reinforcement effect is more obvious after the large pipe shed is added.

(3) The surface settlement caused by ring soil excavation accounts for about $50 \%$ to $60 \%$ of the total settlement value, and the surface settlement caused by core soil and lower bench excavation account for $23 \%$ to $35 \%$ and $15 \%$ to $20 \%$ of the total settlement value, respectively. Annular soil excavation is a key process in the entire construction phase.

(4) After the combined reinforcement measures were taken, the final surface settlement was reduced by $18.5 \mathrm{~mm}$ compared with the absence of reinforcement measures. After the excavation of the annular soil, the surface settlement of the combined reinforcement measures was reduced by $12.7 \mathrm{~mm}$ compared with the surface settlement without the reinforcement measures. The combined reinforcement measures focused on restraining the surface settlement caused by the excavation of the annular soil.

(5) The settlement groove width caused by the excavation of the ultrasmall distance underneath the tunnel is small, and the increase is not obvious with the tunnel excavation. However, the curvature and peak value of the settlement trough is large and prone to collapse. After the reinforcement measures were taken, the settlement groove width did not change significantly, but the curvature and peak value of the settlement trough decreased significantly.

\section{Data Availability}

The data used to support the findings of this study are available from the corresponding author upon request.

\section{Conflicts of Interest}

The authors declare that they have no conflicts of interest regarding the publication of this paper.

\section{Acknowledgments}

The authors are grateful for the financial support from the General Project of Shandong Provincial Natural Science Foundation of China (Grant no. ZR2020ME096).

\section{References}

[1] B. Duan, S. Wu, Y. Zhu et al., "Monitoring measurement and analysis of Jiaohua square station and Jiaoxi section of Harbin Metro," Railway Engineering, no. 1, pp. 36-38, 2013.

[2] Z. Song, X. Tian, Y. Zhang, and S.V. Castorina, "A new modified peck formula for predicting the surface settlement based on stochastic medium theory," Advances in Civil Engineering, vol. 2019, Article ID 7328190, 14 pages, 2019.

[3] Z. Chen, F. Zhou, Y. Wen, L. Huang, and Y Ren, "Research on settlement control standards and techniques of super-shallow tunnels underpassing expressway subgrade," Highway Engineering, pp. 1-16, 2021.

[4] J. Li, Analysis of Ground Deformation Caused by ShallowBuried and Under-Cut Construction of Harbin Metro Tunnel, Xi'an University of Architecture and Technology, Xi'an, China, 2012.

[5] C. Zhang, Y Cai, W. Zhu, and D. Baraldi, "Numerical study and field monitoring of the ground deformation induced by large slurry shield tunnelling in sandy cobble ground," $A d-$ vances in Civil Engineering, vol. 2019, Article ID 4145721, 12 pages, 2019.

[6] L. Ruan and X. Sun, "Study of the influence of new shield tunnel construction on existing railway subgrade," Railway Standard Design, vol. 62, no. 6, pp. 99-104, 2018.

[7] H. Huang, P. Zhou, P. Chen et al., "Theoretical research on different construction methods for double-line highway tunnel under-passing existing railway tunnel," Railway Standard Design, no. 11, pp. 23-26, 2013.

[8] S. Wu, B. Duan, Y. Zhu et al., "Predication of the grouting effect of surrounding rock based on the theory of grey," Journal of Railway Engineering Society, no. 11, pp. 23-26, 2013.

[9] Z. Zhu, S. Huang, and Y. Zhu, "Study of road surface settlement rule and controlled criterion for railway tunnel under crossing highway," Rock and Soil Mechanics, vol. 33, no. 2, pp. 558-563, 2012.

[10] Z. Wang, S. Du, W. Zhang et al., "Analysis of construction settlement of shallow railway tunnel under crossing the highway," Chinese Journal of Underground Space and Engineering, vol. 5, no. 3, pp. 531-535, 2009.

[11] The People's Republic of China Industry Recommended Standards Writing Group, JTG/TD70-2010 Highway Tunnel Design Rules, People's Communications Press, Beijing, China, 2010. 